\title{
Character changes during the early post-embryonic development of the centipede Strigamia maritima (Leach, 1817) (Chilopoda: Geophilomorpha)
}

\author{
Vincent Vedel ${ }^{{ }^{*}} \&$ Wallace Arthur \\ Department of Zoology, School of Natural Sciences, \\ National University of Ireland, Galway, University Road, Galway, Ireland \\ † Present address: UMR ECOFOG, INRA, Campus Agronomique de Kourou, \\ BP 709, 97387 Kourou, French Guiana \\ *Corresponding author. E-mail: vincent.vedel@ecofog.gf
}

\begin{abstract}
For many kinds of ecological, evolutionary and developmental study, it is important to be able to describe the life-history of the individuals of a particular species/population. In the case of myriapods and other arthropods, this involves separating the different life-history stages (or stadia or instars) that are separated by moults. However, it has recently been pointed out that in the earliest post-embryonic stages the cuticle is still quite flexible; this means that visible external developmental changes can occur between moults as well as during them. Here, we provide evidence for such inter-moult changes in the coastal geophilomorph centipede Strigamia maritima. The character states studied enable finer-scale resolution of early post-embryonic forms than was hitherto possible. Specifically, we describe five transitional forms during a period in which just two (Peripatoid and Foetus) have traditionally been recognized.
\end{abstract}

\section{Keywords}

moult, development, arthropod, post-embryonic stage, morphological change, myriapod, centipede, evo-devo

\section{Introduction}

Arthropods, including myriapods, are conventionally thought of as developing, postembryonically, by a series of changes concentrated in moulting events. However, this 
is somewhat untrue for young arthropods, especially just after hatching. Indeed, the cuticle is still soft then, and several morphological structures (appendages, antennae, mouthparts) are developing. As Minelli et al. (2006) have pointed out, many visible changes in early post-embryonic stages occur between moults and not during the moult event itself, which would indicate a continuous development for some morphological characters. In this study, we focused only on external visible characters, because of their use for separating morphological stages. Describing early arthropod stages just by considering moults appears now as incomplete. A more refined description of stages, integrating moults and other less dramatic morphological changes, would be appropriate to get a more complete picture of early post-embryonic growth.

The centipede Strigamia maritima has become a model system for understanding the evolution of segment number in arthropods (Arthur \& Chipman 2005). Studies of its life cycle and ecology (Lewis 1961), gene expression patterns (Kettle et al. 2003), and the plasticity and heritability of its leg-bearing segment (LBS) number (Vedel et al. 2008, Vedel et al. 2009) all employ descriptions of its morphological development. A more complete understanding of early post-embryonic stages is needed for further work on S. maritima, both as a model system in evo-devo and more generally.

\section{The early life history of $S$. maritima}

Lewis (1961) was the first to observe and describe the full life-cycle of $S$. maritima. Females lay eggs just once a year. They are laid at the end of May or the beginning of June, with a remarkable synchronization between different populations. This timing corresponds to the period when eggs are the least likely to be inundated with seawater. Between about five and 25 spherical eggs (around $1 \mathrm{~mm}$ in diameter) are laid in small cavities dug into the substrate. Females coil around the broods and exhibit maternal care behaviour, the main functions of which are probably deterring egg predators, removing fungi and keeping the eggs humid. Eggs (Fig. $1 \mathrm{~A}, \mathrm{~B})$ are generally whitish when just laid, becoming yellowish khaki during their development and eventually turning to pink (due to gut cell pigmentation) a few days before hatching.

By the end of June, about four weeks after being laid, broods hatch (see Fig. 1C). The hatchlings are not yet capable of walking, or indeed of making any significant movements. They cannot feed, the mouthparts are not fully developed, and they still live out of the yolk reserve, situated on the ventral side of the anterior part of the trunk. Hatchlings still remain in the brooding site under maternal protection. This period, which also lasts around four weeks, was first divided into three stages, separated by moulting events (Lewis 1961). Embryos hatch at the last embryonic stage, then moult into the Peripatoid "in which the trunk is of uniform diameter and the limbs better developed", and then moult into the Foetus "capable of writhing movements". The Peripatoid is so named because of its superficial resemblance to the 

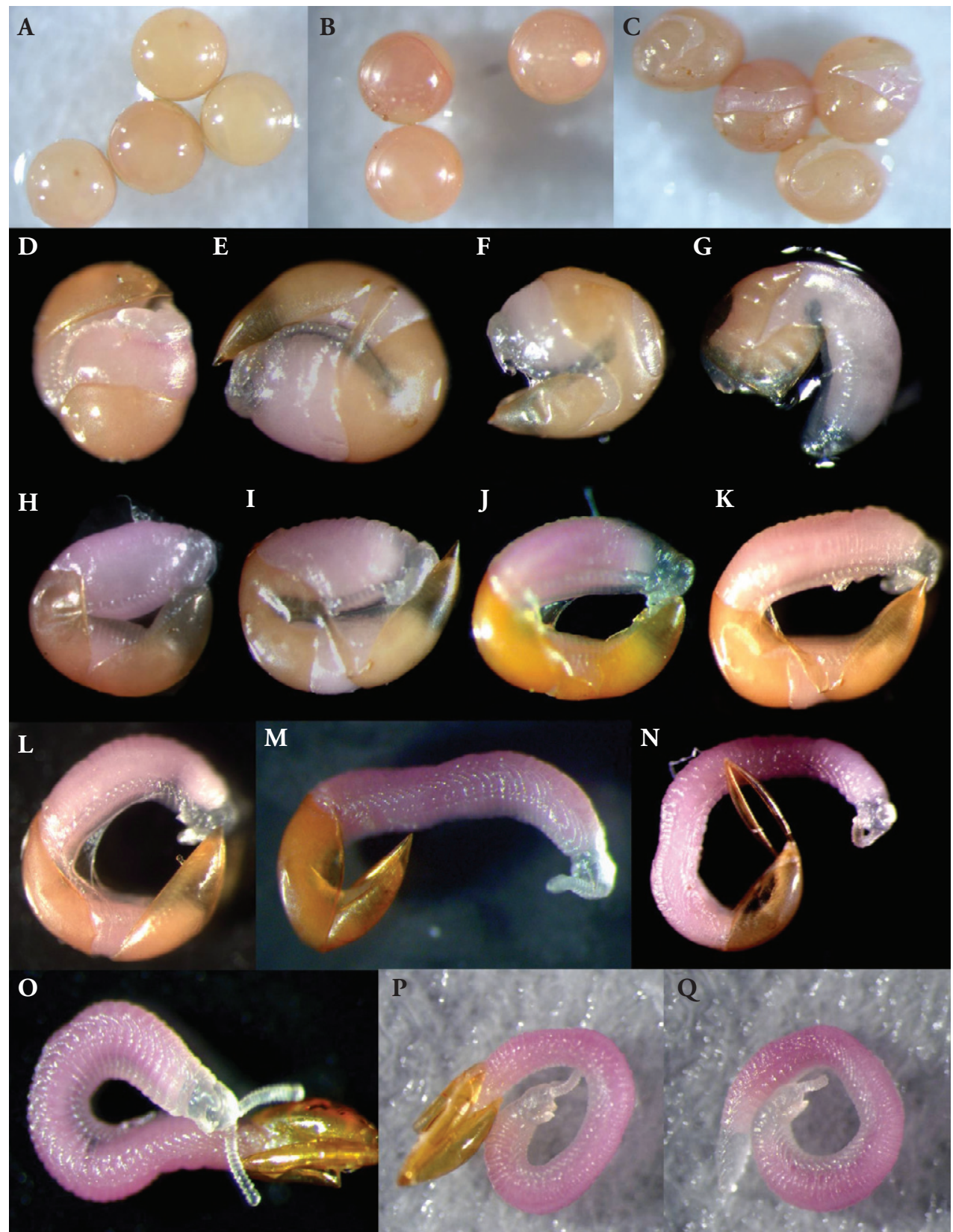

Figure 1. The different stages from recently laid eggs to the early post-embryonic stage Foetus III. Magnification variable - eggs are $1 \mathrm{~mm}$ in diameter; latest stage shown is about $7 \mathrm{~mm}$ in length. A-B, eggs; C-F, Hatchling/Last embryonic stage; G-I, Peripatoid I; J-K, Peripatoid II; L-N, Foetus I; O-P, Foetus II; Q, Foetus III. 
onychophoran Peripatus (Eason 1964). At the end of July, hatchlings moult into the first juvenile stage, Adolescens I. This is the last stage to be brooded by the mother. It is very similar to the adult, with limbs and mouthparts fully developed. After about one month (late August), Adolescens I disperse and begin foraging.

However, as Minelli et al. (2006) have argued, the "current description of arthropod late embryonic and postembryonic development is dominated by a cuticular view", which leads to the fact that this "discontinuous process" hides other uninterrupted mechanisms which occur in the meantime.

\section{Materials and methods}

To refine Lewis's description of early post-embryonic stages (from hatching to the Adolescens I stage) embryos were sampled from a dense $S$. maritima population situated near Brora, north eastern Scotland, which has been described previously (Vedel et al. 2008). Clutches were collected on $13^{\text {th }}$ June 2006 from a stretch of the Brora coastline less than $50 \mathrm{~m}$ long. Five clutches having between 4 and 7 eggs were incubated at $13^{\circ} \mathrm{C}$ from $14^{\text {th }}$ June 2006 until they reached the stage just before being Adolescens I. Live embryos were kept in Petri dishes sandwiched between two layers of Whatmann filter paper soaked in locust embryo saline (LES). Every 24 or 48 hours, soaked filter papers were replaced to keep them in approximately constant humidity and salinity. Dead embryos were removed as soon as possible to avoid any contamination.

Observations of live hatchlings were conducted every 24 hours for almost two months, using a Stemi SV-11 dissecting microscope (Zeiss, Jena, Germany). They were photographed using a Leica DCFC300F digital camera controlled by Firecam 1.2 software (Leica Micro-systems, Cambridge, UK) when a significant morphological change was visible.

Characters chosen for examination (see Table 1) were classified into three categories and were:

A) General body growth:

1) Size of the split in the egg shell

2) Size of the swollen segments where the yolk is stored (LBS 1 to 17)

3) Angle of opening of the full body (becoming straight)

4) Growth in length, body becoming slender and long

5) Shell being stuck to or detached from the body

B) Growth and differentiation of appendages

6) Size, segmentation and orientation of the antennae

7) Shape of the forcipules

8) Size and shape of legs 
C) Movement

9) Movement (writhing and then crawling)

\section{Results}

In the following description, we refer to six "stages", but not all are separated from each other by moults.

1) Hatching/Last embryonic stage: It takes 1 day from the beginning of the opening of the egg shell to a large opening, letting the last embryonic stage show through (Fig. $1 \mathrm{C}-\mathrm{F})$. The anterior end is swollen and full of yolk. The embryo is totally folded. The antennae are poorly developed in size and in differentiation, but are segmented and are stuck to the body. Limbs are not segmented yet and are round buds. The shell covers more than $50 \%$ of the exposed body surface. The body length is around $2 \mathrm{~mm}$. A moult event occurs at the end of this stage (3-4 days; Fig. 1D-F).

2) Peripatoid I: The embryo is folded at LBS 17. The anterior end is less swollen. The antennae have a similar shape although the segments are more visible than in the previous stage and orientated downwards. The shell covers about half of the exposed body surface. The body length is around $3-4 \mathrm{~mm}$ and no movements occur (3-4 days; Fig. 1G-I).

3) Peripatoid II: The embryo is still folded but with an angle of opening of around 30 degrees. There is barely any yolk reserve. The antennae are getting slender and longer and are still orientated downwards. It seems that the articles did not increase since hatching. The forcipules are not distinguishable, but limb buds are growing and extending laterally. The shell covers less than half of the body (around 30-40\%) and is breaking in two parts. The body length is around 4-5 $\mathrm{mm}$. The embryo does not move. A moult event occurs at the end of this stage (5-6 days; Fig. 1J-K).

4) Foetus I: The body is folded at an angle of around 45 degrees or in a circle. The antennae are exactly like in the previous stage, slightly elongated, orientated backwards but with the articles becoming more visible. The forcipules are visible but poorly developed with structures not fully differentiated and immobile. The limb buds start to differentiate, to be a bit more elongated and not round. Segments of limbs start to be visible. They are not moving yet. The shell is separated into two pieces covering the posterior part of the body. The body is becoming slender and longer and measures around $6 \mathrm{~mm}$ with no visible movement (5-6 days; Fig. 1L-N).

5) Foetus II: The body is no longer folded. Antennae are well elongated with articles visible and orientated forwards. They are not stuck to the body any more and are 
capable of movement. The forcipules are longer with structures starting to be differentiated but are still not functional. The limbs are now elongated with segments clearly visible. The shell is attached to the Foetus only at the most posterior part. The ecdysis from the previous stage is still happening in this posterior part, protected by the shell. The body length is around $7 \mathrm{~mm}$, and the animal is capable of writhing movements, especially when reacting to light and/or increase of temperature (3-4 days; Fig. 1O-P).

6) Foetus III: The body is totally free from the shell, and measures around $7 \mathrm{~mm}$ in length. Antennae and forcipules are well developed: they are longer and mobile with structures clearly visible. The limbs are elongated with a visible segmentation and almost mobile (Fig. 1Q). The embryo is capable of writhing movements and can also crawl several $\mathrm{cm}$. This is the last stage before the moult to become Adolescens I (which we kept for 3-5 days, after which they died).

\section{Discussion}

It has been conventional to think of the post-embryonic development of myriapods and other arthropods as being composed of a series of discrete morphological changes, concentrated into moulting events. This is in contrast, of course, to the development of animals without an exoskeleton, where development is generally seen as being continuous. However, as we mentioned in the Introduction, it has recently been pointed out (Minelli et al. 2006) that this distinction is not so clear, especially for early postembryonic stages.

It appears in this study that some characters are continuous while some others are interrupted in their development. The general growth characters (characters 1 to 5), describing the overall body shape, are continuous throughout early development. Therefore there may be more morphological differences between a body shape belonging to an early stage and a body shape belonging to the late same stage than between one from a late stage and another one from the next early stage. Nevertheless the appendages segment and some of their structures (limbs and forcipules) differentiate only during the moult. Elongation and differentiation of those structures, as well as internal development (such as muscle for movement), seem to be continuous.

During the Peripatoid I and Peripatoid II stages no new segments or structures were formed in the limb buds or in the antennae, only their shapes changed due to the elongation of the existing structures. During the three Foetus stages, the forcipules, the antennae and the limbs went from immobile to mobile and took their final position (antennae forward and limbs laterally). In the antennae no segments or structures were added but the existing ones became more extended and elongated. For the legs, it seems that they not only elongated but they also differentiated during the Foetus stages, especially between the Foetus I and Foetus II stages. Between Peripatoid II and Foetus I stages (at the ecdysis), there were no visible 
differences for the antennae. It seems that limb buds produce more limb segments during this moult. The forcipules differentiated also at this point. Segments and articles developed at the moult.

Early post-embryonic stages of centipedes and other myriapods are harder to study than later ones. Relatively few such studies have been carried out. Andersson (1989) studied the post-embryonic development of several lithobiomorph species, though without a particular focus on the early stages. Lewis (1961) did likewise for the geophilomorph species that we are reporting further information on here.

The scarcity of studies on the early post-embryonic development of centipedes is partly due to the difficulty, in most species, of finding eggs in sufficient numbers to follow their post-hatching progress. Geophilomorphs are particularly difficult in this respect because it has so far proved impossible to breed them in the lab. 'Inland' species, inhabiting for example woodland or moorland, occur at relatively low density in the field, and clutches of eggs from these species are hard to find. In contrast, $S$. maritima reaches very high densities (up to 100 per square metre, personal observation), and, in some (but by no means all) populations, hundreds of eggs can be collected in a single day during the breeding season from an area of just a few square metres of prime habitat.

This availability of large numbers of eggs has enabled both Lewis (1961) and ourselves to document the post-embryonic development of $S$. maritima. The advances we report here over Lewis's earlier descriptions are twofold: first, the use of colour photomicrography rather than drawing; and second, the detection of changes occurring within stages, notably the Peripatoid and Foetus. We believe that our Figure 1 constitutes the most complete photographic record to date of the early post-embryonic stages of any of the 1000+ species of geophilomorph centipedes.

We must, however, emphasize the probabilistic rather than absolute nature of the between-moult changes. For example, what we call here Foetus I and II are distinguished by six features (see Table 1). However, not all individuals will exhibit exactly the same combination of changes. Some of the characters are inherently variable, such as the length of time taken for the antennae to become 'unstuck' from the body and to point forwards. Typically, this happens along with the other five changes, but in some individuals it will happen a little earlier or later.

Finally, we should also note that we tried to obtain better resolution of the later post-embryonic stages (from Adolescens I to Maturus Senior, data unpublished) but were unable to improve on the degree of statistical separation obtained by Lewis (1961). Indeed, while our Adolescens I individuals were very distinct from the later stages (as were his), our later stages appeared to overlap with each other more than his did in the same characters. We are not sure if this represents a real difference between the populations studied, but this is possible given the extent of geographic separation, with our populations being from northern Scotland, his from southern England; a separation of several hundred kilometers. Lewis (1962) already found differences between a population in Plymouth (Devon), in which only four post-embryonic stages could be distinguished, and his original study population in Sussex. 


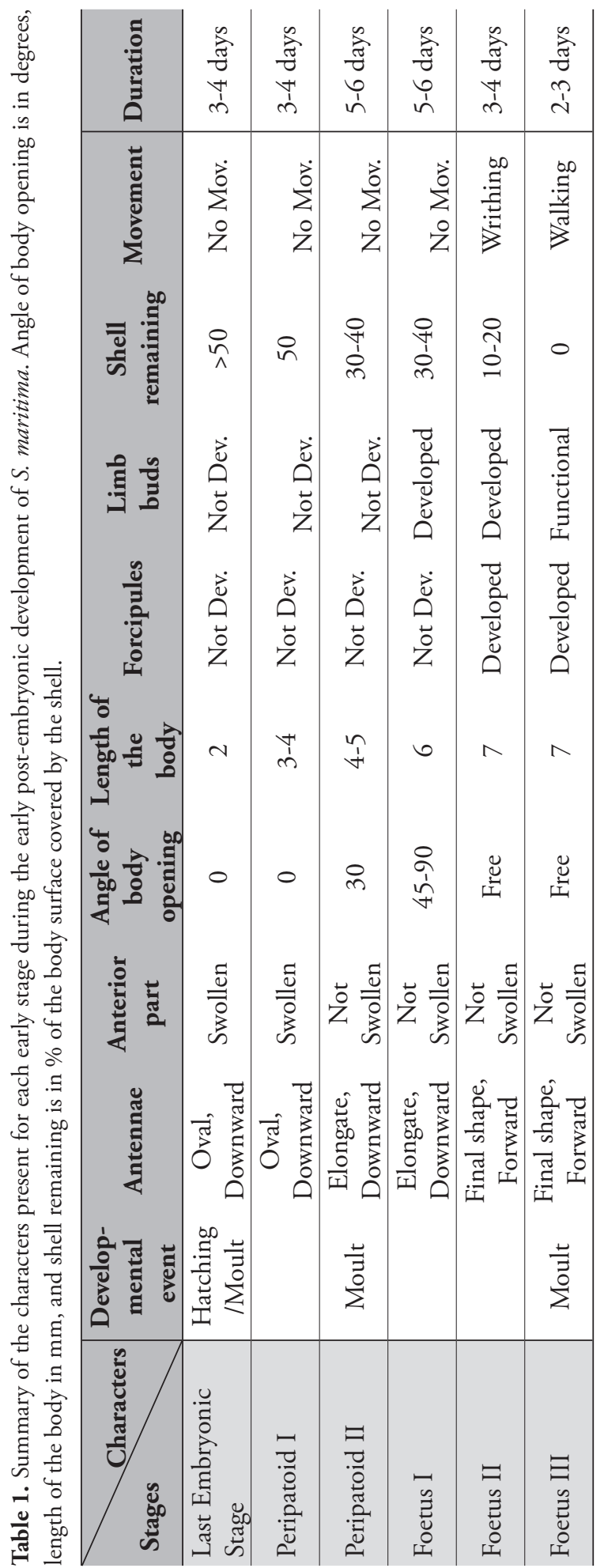




\section{Acknowledgements}

The authors would like to thank the two anonymous reviewers for their constructive and helpful comments on the manuscript. This work was supported by the D'Arcy Thompson Fellowship of the National University of Ireland (to WA).

\section{References}

Andersson, G. (1989) About the duration of the different stadia in the post-embryonic development of some lithobiomorph centipedes. - In: Minelli, A. (ed.) Proceedings of the $7^{\text {th }}$ International Congress of Myriapodology, EJ Brill, Leiden, 323-335.

Arthur, W., Chipman, A.D. (2005) The centipede Strigamia maritima: what it can tell us about the development and evolution of segmentation. - BioEssays 27: 653-660.

Arthur, W., Kettle, C. (2001) Geographic patterning of variation in segment number in geophilomorph centipedes: clines and speciation. - Evolution and Development 3: 34-40.

Eason, E.H. (1964) Centipedes of the British Isles. Frederick Warne \& Co. Ltd., London and New York, x+294 pp.

Kettle, C., Johnstone, J., Jowett, T., Arthur, H. \& Arthur, W. (2003) The pattern of segment formation, as revealed by engrailed expression, in a centipede with a variable number of segments. - Evolution and Development 5: 198-207.

Lewis, J.G.E. (1961) The life history and ecology of the littoral centipede Strigamia (Scolioplanes) maritima (Leach). - Proceedings of the Zoological Society of London 137: 221-248.

Lewis, J.G.E. (1962) The ecology, distribution and taxonomy of the centipedes found on the shore in the Plymouth area. - Journal of the Marine Biological Association of the U.K. 42: 655-664.

Minelli, A., Brena, C., Deflorian, G., Maruzzo, D. \& Fusco, G. (2006) From embryo to adult - beyond the conventional periodization of arthropod development. - Development Genes and Evolution 216: 373-383.

Vedel, V., Chipman, A.D., Akam, M. \& Arthur, W. (2008) Temperature-dependent plasticity of segment number in an arthropod species: the centipede Strigamia maritima. - Evolution and Development 10: 488-493.

Vedel, V., Brena, C. \& Arthur, W. (2009). Demonstration of a heritable component of the variation in segment number in the centipede Strigamia maritima. - Evolution and Development 11: 435-441. 
